Proyecciones Journal of Mathematics

Vol. 31, No 3, pp. 299-308, Sepember 2012.

Universidad Católica del Norte

Antofagasta - Chile

\title{
Double lacunary sequence spaces of double sequence of interval numbers
}

\author{
AYHAN ESI \\ Adiyaman University, Turkey \\ Received : January 2012. Accepted : August 2012
}

\begin{abstract}
In this paper we introduce the concepts of double lacunary strongly convergence and double lacunary statistical convergence of double interval numbers. We prove some inclusion relations and study some of their properties.
\end{abstract}

Subjclass [2000] : 40C05,40J05, 46A45.

Keywords : Sequence space, Lacunary sequence, interval numbers. 


\section{Introduction}

Interval arithmetic was first suggested by Dwyer [12] in 1951. Development of interval arithmetic as a formal system and evidence of its value as a computational device was provided by Moore [15] in 1959 and Moore and Yang [16] 1962. Furthermore, Moore and others [12], [13], [14], [17] and [18] have developed applications to differential equations.

Chiao in [9] introduced sequence of interval numbers and defined usual convergence of sequences of interval number. Recently Esi $[1-3]$ and Şengönül and Eryilmaz in [11] introduced and studied some sequence spaces of interval numbers.

The idea of statistical convergence for ordinary sequences was introduced by Fast [7] in 1951. Schoenberg [8] studied statistical convergence as a summability method and listed some of elemantary properties of statistical convergence. Both of these authors noted that if bounded sequence is statistically convergent, then it is Cesaro summable. Existing work on statistical convergence appears to have been restricted to real or complex sequence, but several authors extended the idea to apply to sequences of fuzzy numbers and also introduced and discussed the concept of statistically sequences of fuzzy numbers.

\section{Preliminaries}

A double sequence of real numbers is a function $x: \mathbf{N} \times \mathbf{N} \rightarrow \mathbf{R}$. We shall use the notation $x=\left(x_{k, l}\right)$.

A double sequence $x=\left(x_{k, l}\right)$ has a Pringsheim limit $L$ (denoted by $P-\lim x=L)$ provided that given an $\varepsilon>0$ there exists an $N \in \mathbf{N}$ such that $\left|x_{k, l}-L\right|<\varepsilon$ whenever $k, l>N$. We shall describe such an $x=\left(x_{k, l}\right)$ more briefly as "P - convergent" [4]. The double sequence $x=\left(x_{k, l}\right)$ is bounded if there exists a positive number M such that $\left|x_{k, l}\right|<M$ for all $k$ and $l$,

$$
\|x\|=\sup _{k, l}\left|x_{k, l}\right|<\infty .
$$

Let $p=\left(p_{k, l}\right)$ be a double sequence of positive real numbers. If $0<h=$ $\inf _{k, l} p_{k, l} \leq p_{k, l} \leq H=\sup _{k, l} p_{k, l}<\infty$ and $D=\max \left(1,2^{H-1}\right)$, then for all $a_{k, l}, b_{k, l} \in \mathbf{C}$ for all $k, l \in \mathbf{N}$, we have

$$
\left|a_{k, l}+b_{k, l}\right|^{p_{k, l}} \leq D\left(\left|a_{k, l}\right|^{p_{k, l}}+\left|b_{k, l}\right|^{p_{k, l}}\right) .
$$

We should note that in contrast to the case for single sequences, a convergent double sequence need not be bounded.The concept of statistical 
convergence was introduced by Fast [7] in 1951. A sequence $x=\left(x_{k}\right)$ is said to be statistically convergent to the number $L$ if for every $\varepsilon>0$

$$
\lim _{n} \frac{1}{n}\left|\left\{k \leq n: \quad\left|x_{k}-L\right| \geq \varepsilon\right\}\right|=0,
$$

where the vertical bars indicate the number of elements in the enclosed set. Later, Mursaleen and Edely [10] defined the statistical analogue for double sequence $x=\left(x_{k, l}\right)$ as follows: A real double sequence $x=\left(x_{k, l}\right)$ is said to be $P$ - statistical convergence to $L$ provided that for each $\varepsilon>0$

$$
P-\lim _{m, n} \frac{1}{m n}\left|\left\{(k, l): k<m, l<n ;\left|x_{k, l}-L\right| \geq \varepsilon\right\}\right|=0 .
$$

In this case, we write $S t_{2}-\lim _{k, l} x_{k, l}=L$ and we denote the set of all $P$ - statistical convergent double sequences by $S t_{2}$.

By a lacunary $\theta=\left(k_{r}\right) ; r=0,1,2, \ldots$ where $k_{o}=0$, we shall mean an increasing sequence of non-negative integers with $h_{r}=k_{r}-k_{r-1} \rightarrow \infty$ as $r \rightarrow$ $\infty$. The intervals determined by $\theta$ will be denoted by $I_{r}=\left(k_{r-1}, k_{r}\right]$. The ratio $\frac{k_{r}}{k_{r-1}}$ will be denoted by $q_{r}$. The space of lacunary strongly convergent sequence space $N_{\theta}$ was defined by Freedman et.al. [5] as follows:

$$
N_{\theta}=\left\{x=\left(x_{k}\right): \lim _{r} \frac{1}{h_{r}} \sum_{k \in I_{r}}\left|x_{k}-L\right|=0, \text { for some } L\right\} .
$$

The double sequence $\theta_{r, s}=\left\{\left(k_{r}, l_{s}\right)\right\}$ is called double lacunary sequence if there exist two increasing sequences of integers such that

$$
k_{o}=0, h_{r}=k_{r}-k_{r-1} \rightarrow \infty \text { as } r \rightarrow \infty
$$

and

$$
l_{o}=0, \overline{h_{s}}=l_{s}-l_{s-1} \rightarrow \infty \text { as } s \rightarrow \infty .
$$

Notations: $k_{r, s}=k_{r} l_{s}, h_{r, s}=h_{r} \overline{h_{s}}$ and $\theta_{r, s}$ is determined by

$$
\begin{gathered}
I_{r, s}=\left\{(k, l): k_{r-1}<k \leq k_{r} \text { and } l_{s-1}<l \leq l_{s}\right\}, \\
q_{r}=\frac{k_{r}}{k_{r-1}}, \overline{q_{s}}=\frac{l_{s}}{l_{s-1}} \text { and } q_{r, s}=q_{r} \overline{q_{s}} .[6]
\end{gathered}
$$


The set of all double lacunary sequences denoted by $N_{\theta_{r, s}}$ and defined by Savaş and Patterson [6] as follows:

$$
N_{\theta_{r, s}}=\left\{x=\left(x_{k, l}\right): P-\lim _{r, s} \frac{1}{h_{r, s}} \sum_{(k, l) \in I_{r, s}}\left|x_{k, l}-L\right|=0 \text {, for some L }\right\} \text {. }
$$

We denote the set of all real valued closed intervals by IR. Any elements of IR is called interval number and denoted by $\bar{x}=\left[x_{l}, x_{r}\right]$. Let $x_{l}$ and $x_{r}$ be first and last points of $\bar{x}$ interval number, respectively. For $\bar{x}_{1}, \bar{x}_{2} \in \mathbf{I R}$, we have

$\bar{x}_{1}=\bar{x}_{2} \Leftrightarrow x_{1_{l}}=x_{2_{l}}, x_{1_{r}}=x_{2_{r}} . \bar{x}_{1}+\bar{x}_{2}=\left\{x \in \mathbf{R}: x_{1_{l}}+x_{2_{l}} \leq x \leq x_{1_{r}}+x_{2_{r}}\right\}$, and if $\alpha \geq 0$, then $\alpha \bar{x}=\left\{x \in \mathbf{R}: \alpha x_{1_{l}} \leq x \leq \alpha x_{1_{r}}\right\}$ and if $\alpha<0$, then $\alpha \bar{x}=\left\{x \in \mathbf{R}: \alpha x_{1_{r}} \leq x \leq \alpha x_{1_{l}}\right\}$,

$$
\begin{gathered}
\bar{x}_{1} \cdot \bar{x}_{2} \\
=\left\{x \in \mathbf{R}: \min \left\{x_{1_{l}} \cdot x_{2_{l}}, x_{1_{l}} \cdot x_{2_{r}}, x_{1_{r}} \cdot x_{2_{l}}, x_{1_{r}} \cdot x_{2_{r}}\right\} \leq x\right. \\
\leq \max \left\{x_{1_{l}} \cdot x_{2_{l}}, x_{1_{l}} \cdot x_{2_{r}}, x_{1_{r}} \cdot x_{2_{l}}, x_{1_{r}} \cdot x_{2_{r}}\right\} .
\end{gathered}
$$

The set of all interval numbers IR is a complete metric space defined by

$$
d\left(\bar{x}_{1}, \bar{x}_{2}\right)=\max \left\{\left|x_{1_{l}}-x_{2_{l}}\right|,\left|x_{1_{r}}-x_{2_{r}}\right|\right\} \quad[15] .
$$
R.

In the special case $\bar{x}_{1}=[a, a]$ and $\bar{x}_{2}=[b, b]$, we obtain usual metric of

Now we give the definition of convergence of interval numbers:

Definition 1.1. [9] A sequence $\bar{x}=\left(\bar{x}_{k}\right)$ of interval numbers is said to be convergent to the interval number $\bar{x}_{o}$ if for each $\varepsilon>0$ there exists a positive integer $k_{o}$ such that $d\left(\bar{x}_{k}, \bar{x}_{o}\right)<\varepsilon$ for all $k \geq k_{o}$ and we denote it by $\lim _{k} \bar{x}_{k}=\bar{x}_{o}$.

Thus, $\lim _{k} \bar{x}_{k}=\bar{x}_{o} \Leftrightarrow \lim _{k} x_{k_{l}}=x_{o_{l}}$ and $\lim _{k} x_{k_{r}}=x_{o_{r}}$.

Let's define transformation $\bar{x}$ from $\mathbf{N} \times \mathbf{N}$ to IR by $k, l \rightarrow \bar{x}(k, l)=\bar{x}_{k, l}$. We shall use the notation $\bar{x}=\left(\bar{x}_{k, l}\right)$. Then $\bar{x}=\left(\bar{x}_{k, l}\right)$ is called double sequence of interval numbers. The $\bar{x}_{k, l}$ is called $(k, l)^{t h}$ term of sequence $\bar{x}=\left(\bar{x}_{k, l}\right)$.

In this paper, we introduce and study the concepts of double lacunary strongly convergence and double lacunary statistically convergence for interval numbers. 


\section{Main Results}

In this section we give some definition and prove the results of this paper.

Definition 3.1. Let $\theta_{r, s}=\left\{\left(k_{r}, l_{s}\right)\right\}$ be a double lacunary sequence and $p=\left(p_{k, l}\right)$ be any double sequence of strictly positive real numbers. A double sequence $\bar{x}=\left(\bar{x}_{k, l}\right)$ of interval numbers is said to be double lacunary strongly convergent if there is a double interval number $\bar{x}_{o}$ such that

$$
P-\lim _{r, s} \frac{1}{h_{r, s}} \sum_{k \in I_{r, s}}\left[d\left(\bar{x}_{k, l}, \bar{x}_{o}\right)\right]^{p_{k, l}}=0 .
$$

In this case we write $\bar{x}_{k, l} \rightarrow \bar{x}_{o}\left({ }_{2} \bar{N}_{\theta_{r, s}}^{p}\right)$ or ${ }_{2} \bar{N}_{\theta}^{p}-\lim \bar{x}_{k, l}=\bar{x}_{o}$. We denote with ${ }_{2} \bar{N}_{\theta_{r, s}}^{p}$ the set of all lacunary strongly convergent double sequences of interval numbers. In the special case $\theta_{r, s}=\left\{\left(2^{r}, 2^{s}\right)\right\}$, we shall write ${ }_{2} \bar{N}^{p}$ instead of ${ }_{2} \bar{N}_{\theta_{r, s}}^{p}$.

Definition 3.2. Let $\theta_{r, s}=\left\{\left(k_{r}, l_{s}\right)\right\}$ be a double lacunary sequence. A double sequence $\bar{x}=\left(\bar{x}_{k, l}\right)$ of interval numbers is said to be double lacunary statistically convergent to interval number $\bar{x}_{o}$ if for every $\varepsilon>0$

$$
P-\lim _{r, s} \frac{1}{h_{r, s}}\left|\left\{(k, l) \in I_{r, s}: d\left(\bar{x}_{k, l}, \bar{x}_{o}\right) \geq \varepsilon\right\}\right|=0 .
$$

In this case we write $\bar{x}_{k, l} \rightarrow \bar{x}_{o}\left(\bar{s}_{\theta_{r, s}}\right)$ or $\bar{s}_{\theta_{r, s}}-\lim \bar{x}_{k, l}=\bar{x}_{o}$. The set of all double lacunary statistically convergent sequences of interval number is denoted by $\bar{s}_{\theta_{r, s}}$. In the special case $\theta_{r, s}=\left\{\left(2^{r}, 2^{s}\right)\right\}$, we shall write $\bar{s}$ instead of $\bar{s}_{\theta_{r, s}}$.

Theorem 3.1. Let $\bar{x}=\left(\bar{x}_{k, l}\right)$ and $\bar{y}=\left(\bar{y}_{k, l}\right)$ be double sequences of interval numbers.

(i) If $\bar{s}_{\theta_{r, s}}-\lim \bar{x}_{k, l}=\bar{x}_{o}$ and $\alpha \in \mathbf{R}$, then $\bar{s}_{\theta_{r, s}}-\lim \alpha \bar{x}_{k, l}=\alpha \bar{x}_{o}$.

(ii) If $\bar{s}_{\theta_{r, s}}-\lim \bar{x}_{k, l}=\bar{x}_{o}$ and $\bar{s}_{\theta_{r, s}}-\lim \bar{y}_{k, l}=\bar{y}_{o}$, then $\bar{s}_{\theta_{r, s}}-\lim \left(\bar{x}_{k, l}+\bar{y}_{k, l}\right)=$ $\bar{x}_{o}+\bar{y}_{o}$.

Proof. (i) Let $\alpha \in=|\alpha| d\left(\bar{x}_{k, l}, \bar{x}_{o}\right)$. For a given $\varepsilon>0$

$$
\frac{1}{h_{r, s}}\left|\left\{(k, l) \in I_{r, s}: d\left(\alpha \bar{x}_{k, l}, \alpha \bar{x}_{o}\right) \geq \varepsilon\right\}\right|
$$




$$
=\frac{1}{h_{r, s}}\left|\left\{(k, l) \in I_{r, s}: d\left(\bar{x}_{k, l}, \bar{x}_{o}\right) \geq \frac{\varepsilon}{|\alpha|}\right\}\right| .
$$

Hence $\bar{s}_{\theta_{r, s}}-\lim \alpha \bar{x}_{k, l}=\alpha \bar{x}_{o}$.

(ii) Suppose that $\bar{s}_{\theta_{r, s}}-\lim \bar{x}_{k, l}=\bar{x}_{o}$ and $\bar{s}_{\theta_{r, s}}-\lim \bar{y}_{k, l}=\bar{y}_{o}$. We have

$$
\begin{gathered}
d\left(\bar{x}_{k, l}+\bar{y}_{k, l}, \bar{x}_{o}+\bar{y}_{o}\right) \\
\leq d\left(\bar{x}_{k, l}, \bar{x}_{o}\right)+d\left(\bar{y}_{k, l}, \bar{y}_{o}\right) .
\end{gathered}
$$

Therefore given $\varepsilon>0$, we have

$$
\begin{gathered}
\frac{1}{h_{r, s}}\left|\left\{(k, l) \in I_{r, s}: d\left(\bar{x}_{k, l}+\bar{y}_{k, l}, \bar{x}_{o}+\bar{y}_{o}\right) \geq \varepsilon\right\}\right| \\
\leq \frac{1}{h_{r, s}}\left|\left\{(k, l) \in I_{r, s}: d\left(\bar{x}_{k, l}, \bar{x}_{o}\right)+d\left(\bar{y}_{k, l}, \bar{y}_{o}\right) \geq \varepsilon\right\}\right| \\
\leq \frac{1}{h_{r, s}}\left|\left\{(k, l) \in I_{r, s}: d\left(\bar{x}_{k, l}, \bar{x}_{o}\right) \geq \frac{\varepsilon}{2}\right\}\right|+\frac{1}{h_{r, s}}\left|\left\{(k, l) \in I_{r, s}: d\left(\bar{y}_{k, l}, \bar{y}_{o}\right) \geq \frac{\varepsilon}{2}\right\}\right| .
\end{gathered}
$$

Thus, $\bar{s}_{\theta_{r, s}}-\lim \left(\bar{x}_{k, l}+\bar{y}_{k, l}\right)=\bar{x}_{o}+\bar{y}_{o}$.

Theorem 3.2.Let $\theta_{r, s}=\left\{\left(k_{r}, l_{s}\right)\right\}$ be a double lacunary sequence and $\bar{x}=\left(\bar{x}_{k, l}\right)$ be a double sequence of interval numbers. Then

(i) $\bar{x}_{k, l} \rightarrow \bar{x}_{o}\left(\bar{N}_{\theta_{r, s}}^{p}\right)$ implies $\bar{x}_{k, l} \rightarrow \bar{x}_{o}\left(\bar{s}_{\theta_{r, s}}\right)$,

(ii) $\bar{x}=\left(\bar{x}_{k, l}\right) \in \bar{m}$ and $\bar{x}_{k, l} \rightarrow \bar{x}_{o}\left(\bar{s}_{\theta_{r, s}}\right)$ imply $\bar{x}_{k, l} \rightarrow \bar{x}_{o}\left(\bar{N}_{\theta_{r, s}}^{p}\right)$,

(iii) If $\bar{x}=\left(\bar{x}_{k, l}\right) \in \bar{m}$, then $\bar{x}_{k, l} \rightarrow \bar{x}_{o}\left(\bar{N}_{\theta_{r, s}}^{p}\right)$ if and only if $\bar{x}_{k, l} \rightarrow$ $\bar{x}_{o}\left(\bar{s}_{\theta_{r, s}}\right)$, where $\bar{m}=\left\{\bar{x}=\left(\bar{x}_{k, l}\right): \sup _{k, l} d\left(\bar{x}_{k, l}, \bar{x}_{o}\right)<\infty\right\}$.

Proof. (i) Let $\varepsilon>0$ and $\bar{x}_{k, l} \rightarrow \bar{x}_{o}\left(\bar{N}_{\theta_{r, s}}^{p}\right)$. Then we write

$$
\left|\left\{(k, l) \in I_{r, s}: d\left(\bar{x}_{k, l}, \bar{x}_{o}\right) \geq \varepsilon\right\}\right| \leq \sum_{(k, l) \in I_{r, s} d\left(\bar{x}_{k, l}, \bar{x}_{o}\right) \geq \varepsilon} d\left(\bar{x}_{k, l}, \bar{x}_{o}\right)
$$

and

$$
P-\lim _{r, s} \frac{1}{h_{r, s}} \sum_{k \in I_{r, s}}\left[d\left(\bar{x}_{k, l}, \bar{x}_{o}\right)\right]^{p_{k, l}}=0 .
$$

This implies that

$$
P-\lim _{r, s} \frac{1}{h_{r, s}}\left|\left\{(k, l) \in I_{r, s}: d\left(\bar{x}_{k, l}, \bar{x}_{o}\right) \geq \varepsilon\right\}\right|=0 .
$$


This completes the proof (i).

(ii) Suppose that $\bar{x}=\left(\bar{x}_{k, l}\right) \in \bar{m}$ and $\bar{x}_{k, l} \rightarrow \bar{x}_{o}\left(\bar{s}_{\theta_{r, s}}\right)$. Since $\bar{x}=\left(\bar{x}_{k, l}\right) \in$ $\bar{m}$, there is a constant $C>0$ such that $d\left(\bar{x}_{k, l}, \bar{x}_{o}\right) \leq C$. Given $\varepsilon>0$, we have

$$
\begin{gathered}
\frac{1}{h_{r, s}} \sum_{(k, l) \in I_{r, s}}\left[d\left(\bar{x}_{k, l}, \bar{x}_{o}\right)\right]^{p_{k}} \\
=\frac{1}{h_{r, s}} \sum_{d\left(\bar{x}_{k, l}, \bar{x}_{o}\right) \geq \varepsilon(k, l) \in I_{r, s}}\left[d\left(\bar{x}_{k}, \bar{x}_{o}\right)\right]^{p_{k}}+\frac{1}{h_{r, s}} \sum_{d\left(\bar{x}_{k, l}, \bar{x}_{o}\right)<\varepsilon(k, l) \in I_{r, s}}\left[d\left(\bar{x}_{k}, \bar{x}_{o}\right)\right]^{p_{k}} \\
\leq \frac{1}{h_{r, s}} \sum_{d\left(\bar{x}_{k, l}, \bar{x}_{o}\right) \geq \varepsilon(k, l) \in I_{r, s}} \max \left(C^{h}, C^{H}\right)+\frac{1}{h_{r, s}} \sum_{d\left(\bar{x}_{k, l}, \bar{x}_{o}\right)<\varepsilon(k, l) \in I_{r, s}} \varepsilon^{p_{k}} \\
\leq \max \left(C^{h}, C^{H}\right) \frac{1}{h_{r, s}}\left|\left\{(k, l) \in I_{r, s}: d\left(\bar{x}_{k, l}, \bar{x}_{o}\right) \geq \varepsilon\right\}\right|+\max \left(\varepsilon^{h}, \varepsilon^{H}\right) .
\end{gathered}
$$

Thus we obtain $\bar{x}_{k, l} \rightarrow \bar{x}_{o}\left(\bar{N}_{\theta_{r, s}}^{p}\right)$.

(iii) It follows from (i) and (ii).

Theorem 3.3. Let $\theta_{r, s}=\left\{\left(k_{r}, l_{s}\right)\right\}$ be a double lacunary sequence and $\bar{x}=\left(\bar{x}_{k, l}\right)$ be a double sequence of interval numbers. Then

(i) For $\liminf { }_{r} q_{r}>1$ and $\liminf _{s} \bar{q}_{s}>1$ then $\bar{x}_{k, l} \rightarrow \bar{x}_{o}(\bar{s})$ implies $\bar{x}_{k, l} \rightarrow$ $\bar{x}_{o}\left(\bar{s}_{\theta_{r, s}}\right)$,

(ii) For $\lim \sup _{r} q_{r}<\infty$ and $\operatorname{lim\operatorname {sup}_{s}} \bar{q}_{s}<\infty$ then $\bar{x}_{k, l} \rightarrow \bar{x}_{o}\left(\bar{s}_{\theta_{r, s}}\right)$ implies $\bar{x}_{k, l} \rightarrow \bar{x}_{o}(\bar{s})$,

(iii) If $1<\liminf \sin _{r, s} q_{r, s} \leq \operatorname{lim\operatorname {sup}_{r,s}} \bar{q}_{r, s}<\infty$, then $\bar{x}_{k, l} \rightarrow \bar{x}_{o}(\bar{s})$ if and only if $\bar{x}_{k, l} \rightarrow \bar{x}_{o}\left(\bar{s}_{\theta_{r, s}}\right)$.

Proof. (i) Suppose that $\liminf _{r} q_{r}>1$ and $\liminf _{s} \bar{q}_{s}>1$ then there exists a $\delta>0$ such that $q_{r} \geq 1+\delta, \bar{q}_{s} \geq 1+\delta$ for sufficiently large $r$ and $s$ which implies

$$
\frac{h_{r}}{k_{r}} \geq \frac{\delta}{1+\delta} \text { and } \frac{\bar{h}_{s}}{l_{s}} \geq \frac{\delta}{1+\delta} .
$$

Since $h_{r, s}=k_{r} l_{s}-k_{r} l_{s-1}-k_{r-1} l_{s}-k_{r-1} l_{s-1}$ we granted the following

$$
\frac{k_{r} l_{s}}{h_{r, s}} \leq \frac{(1+\delta)^{2}}{\delta^{2}} \text { and } \frac{k_{r-1} l_{s-1}}{h_{r, s}} \leq \frac{1}{\delta}
$$


Now, let $\bar{x}_{k, l} \rightarrow \bar{x}_{o}(\bar{s})$. We are going to prove $\bar{x}_{k, l} \rightarrow \bar{x}_{o}\left(\bar{s}_{\theta_{r, s}}\right)$. Then for sufficiently large $r$ and $s$, we have

$$
\begin{gathered}
\frac{1}{k_{r} l_{s}} \mid\left\{(k, l) \in I_{r, s} ; k \leq k_{r} \text { and } l \leq l_{s}: d\left(\bar{x}_{k, l}, \bar{x}_{o}\right) \geq \varepsilon\right\} \mid \\
\geq \frac{1}{k_{r} l_{s}}\left|\left\{(k, l) \in I_{r, s}: d\left(\bar{x}_{k, l}, \bar{x}_{o}\right) \geq \varepsilon\right\}\right| \\
\geq \frac{(1+\delta)^{2}}{\delta^{2}} \cdot \frac{1}{h_{r, s}}\left|\left\{(k, l) \in I_{r, s}: d\left(\bar{x}_{k, l}, \bar{x}_{o}\right) \geq \varepsilon\right\}\right| .
\end{gathered}
$$

Hence $\bar{x}_{k, l} \rightarrow \bar{x}_{o}\left(\bar{s}_{\theta_{r, s}}\right)$.

(ii) If $\lim \sup _{r} q_{r}<\infty$ and $\lim \sup _{s} \bar{q}_{s}<\infty$ then there exists $C>0$ such that $q_{r}<C$ and $\bar{q}_{s}<C$ for all $r, s \geq 1$. Let $\bar{x}_{k, l} \rightarrow \bar{x}_{o}\left(\bar{s}_{\theta_{r, s}}\right)$ and $\varepsilon>0$. Then there exist $r_{o}<0$ and $s_{o}>0$ such that for every $i \geq r_{o}$ and $j \geq s_{o}$

$$
B_{i, j}=\frac{1}{h_{i, j}}\left|\left\{(k, l) \in I_{i, j}: d\left(\bar{x}_{k, l}, \bar{x}_{o}\right) \geq \varepsilon\right\}\right|<\varepsilon .
$$

Let $M=\max \left\{B_{i, j}: 1 \leq i \leq r_{o}\right.$ and $\left.1 \leq j \leq s_{o}\right\}$ and $\mathrm{m}$ and $\mathrm{n}$ be such that $k_{r-1}<m \leq k_{r}$ and $l_{s-1}<n \leq l_{s}$. Thus we obtain the following

$$
\begin{aligned}
& \frac{1}{m n} \mid\left\{(k, l) \in I_{i, j} ; k \leq m \text { and } l \leq n: d\left(\bar{x}_{k, l}, \bar{x}_{o}\right) \geq \varepsilon\right\} \mid \\
\leq & \frac{1}{k_{r-1} l_{s-1}} \mid\left\{(k, l) \in I_{i, j} ; k \leq k_{r} \text { and } l \leq l_{s}: d\left(\bar{x}_{k, l}, \bar{x}_{o}\right) \geq \varepsilon\right\} \mid \\
\leq & \frac{1}{k_{r-1} l_{s-1}} \sum_{t, u=1,1}^{r_{o}, s_{o}} h_{t, u} B_{t, u}+\frac{1}{k_{r-1} l_{s-1}} \sum_{\left(r_{o}<t \leq r\right) \cup\left(s_{o}<u \leq s\right)} h_{t, u} B_{t, u} \\
\leq & \frac{M}{k_{r-1} l_{s-1}} \sum_{t, u=1,1}^{r_{o}, s_{o}} h_{t, u}+\frac{1}{k_{r-1} l_{s-1}} \sum_{\left(r_{o}<t \leq r\right) \cup\left(s_{o}<u \leq s\right)} h_{t, u} B_{t, u} \\
\leq & \frac{M k_{r_{o}} l_{s_{o}} r_{o} s_{o}}{k_{r-1} l_{s-1}}+\frac{1}{k_{r-1} l_{s-1}} \sum_{\left(r_{o}<t \leq r\right) \cup\left(s_{o}<u \leq s\right)} h_{t, u} B_{t, u} \\
\leq & \frac{M k_{r_{o}} l_{s_{o}} r_{o} s_{o}}{k_{r-1} l_{s-1}}+\left(\sup _{t \geq r_{o} \cup u \geq s_{o}} B_{t, u}\right) \frac{1}{k_{r-1} l_{s-1}} \sum_{\left(r_{o}<t \leq r\right) \cup\left(s_{o}<u \leq s\right)} h_{t, u}
\end{aligned}
$$




$$
\begin{gathered}
\leq \frac{M k_{r_{o}} l_{s_{o}} r_{o} s_{o}}{k_{r-1} l_{s-1}}+\frac{\varepsilon}{k_{r-1} l_{s-1}} \sum_{\left(r_{o}<t \leq r\right) \cup\left(s_{o}<u \leq s\right)} h_{t, u} \\
\leq \frac{M k_{r_{o}} l_{s_{o}} r_{o} s_{o}}{k_{r-1} l_{s-1}}+\varepsilon C^{2} .
\end{gathered}
$$

Since $k_{r}$ and $l_{s}$ both approach infinity as both $\mathrm{m}$ and $\mathrm{n}$ approach infinity it follows that

$$
\frac{1}{m n} \mid\left\{(k, l) \in I_{i, j} ; k \leq m \text { and } l \leq n: d\left(\bar{x}_{k, l}, \bar{x}_{o}\right) \geq \varepsilon\right\} \mid \rightarrow 0 .
$$

This completes the proof.

(iii) It follows from (i) and (ii).

Acknowledgement: The author is indebted to the referee for his/her helpful suggestions.

\section{References}

[1] A. Esi, Strongly almost $\lambda$-convergence and statistically almost $\lambda$-convergence of interval numbers, Scientia Magna, 7 (2), pp. 117$122,(2011)$.

[2] A. Esi, Lacunary sequence spaces of interval numbers, Thai Journal of Mathematics, 10 (2), pp. 445-451, (2012).

[3] A. Esi, A new class of interval numbers, Journal of Qafqaz University, Mathematics and Computer Science, pp. 98-102, (2012).

[4] A. Pringsheim, Zur theorie der zweifach unendlichen Zahlenfolgen, Mathematische Annalen, 53, pp. 289-321, (1900).

[5] A. R. Freedman, J.J.Sember and M.Raphael, Some Cesaro type summability, Proc.London Math.Soc., 37 (3), pp. 508-520, (1978).

[6] E. Savaş and R.F.Patterson, Lacunary statistical convergence of multiple sequences, Applied Mathematics Letters, 19, pp. 527-534, (2006).

[7] H. Fast, Sur la convergence statistique, Collog.Math.2, pp. 241-244, (1951). 
[8] I. J. Schoenberg, The integrability of certain functions and related summability methods, Amer. Math. Monthly, 66, pp. 361-375, (1959).

[9] Kuo-Ping Chiao, Fundamental properties of interval vector max-norm, Tamsui Oxford Journal of Mathematics, 18 (2), pp. 219-233, (2002).

[10] M. Mursaleen and O.H.Edely, Statistical convergence of double sequences, J. Math. Anal. Appl. 288 (1), pp. 223-231, (2003).

[11] M. Şengönül and A. Eryılmaz, On the sequence spaces of interval numbers, Thai Journal of Mathematics, 8 (3), pp. 503-510, (2010).

[12] P. S. Dwyer, Linear Computation, New York, Wiley, (1951).

[13] P. S. Dwyer, Errors of matrix computation, simultaneous equations and eigenvalues, National Bureu of Standarts, Applied Mathematics Series, 29, pp. 49-58, (1953).

[14] P. S. Fischer, Automatic propagated and round-off error analysis, paper presented at the 13th National Meeting of the Association of Computing Machinary, June (1958).

[15] R. E. Moore, Automatic Error Analysis in Digital Computation, LSMD-48421, Lockheed Missiles and Space Company, (1959).

[16] R. E. Moore and C. T. Yang, Interval Analysis I, LMSD-285875, Lockheed Missiles and Space Company, (1962).

[17] R. E. Moore and C. T. Yang, Theory of an interval algebra and its application to numeric analysis, RAAG Memories II, Gaukutsu Bunken Fukeyu-kai, Tokyo, (1958).

[18] S. Markov, Quasilinear spaces and their relation to vector spaces, Electronic Journal on Mathematics of Computation, 2 (1), (2005).

\author{
Ayhan Esi \\ Adiyaman University, \\ Science and Art Faculty, \\ Department of Mathematics, \\ 02040, Adiyaman, \\ Turkey \\ e-mailaesi23@adiyaman.edu.tr
}

